\title{
El esquema de Kohlberg revisado: R. S. Peters y la educación moral temprana
}

\author{
BEATRIZ FERNÁNDEZ HERRERO \\ Universidad de Santiago de Compostela
}

\begin{abstract}
SUMARY.-1) For his writer, it is not possible to establish a split between reason and passion, since the affectionate aspects of morals are closely linked to the use or reason: one's own reason of assumed moral concepts forms an affectionate reply to other people in society, defined as social fee-
\end{abstract} ling or altruism.

2) As far as the theme of contents, Peters establishes his proposal by replying to the following questions:

A. WHY WORRY ABOUT THE CONTENTS? The author gives two types of reason: social reason: not all adults evolue towards superior states, many remain in the conventional state, in which it is essential to possess moral contents by which to be guided. Individual reason: learning of the contents is a pre-requisite to the application of rules in a autonomous manner.

B. WHAT TYPE OF CONTENTS MUST BE TAUGTH? In reply. Peters elaborates a list of «basic rules», justifiable under any social situation, which along whith principles will constitute the contents of moral education, since according to the author, they are appropriate for whatever form social life the individuals could find.

C. How SHould the CONTENTS Be taught? Peters answers this in a blunt manner: in watever way that may contribute to the children learning rules, taking care not to dull their capacity for developing an independent attitude oppossing them. And as yet the techniques of cognitive stimulation cannot be used whith small children, in the preconventional and conventional states, the author believes that one must employ some form of «instruction».

In short, it can be said that the legitimation of Peters' proposal centres on his consideration of morales as actions, which implies the fact that the child is an agent before being a conscious agent, which allows him to express a series of interrogatives as a way of justification of his support of the teaching in early moral education, since his objective must be not so much the development of «forms» and «models» of reasoning, but rather the improvement of behaviour and moral actions of the subject, paying attention to the worry «How to succeed in making the pupils want to become moral?», a worry that must extend to the educational process from the begining, since the ultimate idea of Peters' system is that early moral education is a task that cannot be given up. 
Desde que Platón, en su Menón, se planteó por primera vez la cuestión de si la virtud era algo innato en el hombre, o bien algo que éste había de adquirir mediante la enseñanza o el ejercicio, el tema de la educación moral ha sido un tema muy abordado en todas las épocas, como lo puede demostrar un repaso por la historia de la educación y de la ética; pero últimamente ha venido adquiriendo un interés cada vez mayor, existiendo múltiples razones para ello basadas en las exigencias de nuestras sociedades actuales, por lo que éste será el objeto de estudio del presente trabajo.

Etimológicamente, «ethos» $\mathrm{y}$ «mos», dos términos equivalentes, se refieren o significan «modo de ser» o «carácter», pero no innato o biológico sino adquirido a lo largo de la existencia del hombre, lo cual presupone la capacidad de éste para ser educado, ya que sólo puede adquirirse a través de la educación.

A la hora de hacer un estudio y de plantear una propuesta acerca del tema de la educación moral, hay que contar, evidentemente, con la psicología, la cual describe de un modo empírico lo que es el desarrollo moral; sin embargo, para todo enfoque serio de este ámbito de la educación, es esencial una noción previa de la «moral», es decir, una teoría moral, puesto que toda explicación acerca de ella ha de hacerse desde un punto de vista ético particular.

De ahí precisamente la importancia y la necesidad de que el filósofo se ocupe del tema de la educación moral, ya que, si la delimitación de la moral no es una cuestión neutral, y si entendemos su objetivo como el logro del máximo desarrollo a este nivel, ha de ser la ética la que nos diga cuál es el planteamiento ideal o el «deber ser» del desarrollo moral, o dicho en otras palabras, qué nivel de desarrollo moral puede ser considerado como más deseable que los demás.

Por todo esto, el presente trabajo se centrará en analizar las críticas y sugerencias que R. S. Peters, como filósofo de la educación, le hace, en el plano teórico, a la teoría psicológica del desarrollo moral de L. Kohlberg.

\section{INTRODUCCIÓN}

Como paso previo para delimitar la situación, podemos empezar por intentar exponer, de un modo rápido, los planteamientos filosófico-morales de las corrientes de educación moral que se han venido planteando en los últimos tiempos:

El primer paradigma del que hay que hablar es el denominado comúnmente LA CORRIENTE CONVENCIONAL. En él se hace una asimilación de la educación moral a un proceso de socialización, en el que el educando ha de interiorizar unos valores y normas sociomorales de su grupo o su sociedad. Educar moralmente es, pues, para esta corriente, presentar a la persona unas actitudes y unas conductas que lo integren en su sociedad, es decir, que permitan que se integre de una manera armoniosa y no discordante dentro de ella. Se trataría, entonces, de la presentación e imposición de unos valores convencionales, basados en la autoridad otorgada por la tradición, de modo que el educando los asimile sin realizar ningún tipo de cuestionamiento respecto a ellos.

Como reacción a este convencionalismo acrítico surge a partir de los años 60 una corriente de relativismo individualista, que sostiene todo lo contrario que la co- 
rriente tradicional o convencional: los valores no han de estar determinados por la sociedad, sino que ha de ser cada persona, cada individuo, el que decida por sí mismo cuáles han de ser sus deberes morales; esta corriente se materializa sobre todo en el movimiento de la CLARIFICACIÓN DE VALORES, que, pese a que favorece el desarrollo de la tolerancia y la reflexión sobre los intereses de uno mismo, suscita críticas en cuanto a que no distingue entre los valores morales y los de otro tipo, y que no se apoya en ninguna teoría de educación moral.

Frente a los relativismos en que caen las dos corrientes anteriores -un relativismo cultural por el trasfondo convencionalista del modelo tradicional, y un relativismo individualista en la clarificación de valores, al rechazar toda afirmación de valores interindividuales - surge el ENFOQUE COGNITIVO-EVOLUTIVO como propuesta alternativa a ambos. A pesar de que todos sus representantes comparten los aspectos esenciales del enfoque (como puede ser la consideración del carácter racional de la moralidad), éste se concreta de una manera específica en cada uno de ellos, entre los que se encuentran L. Kohlberg en el área psicológica del desarrollo del juicio moral, y R. S. Peters en el campo teórico de la educación moral.

Como herederos de la moral kantiana, de carácter formalista y racionalista, todos ellos coinciden en señalar como idea central de este enfoque la propuesta de una educación moral basada en los principios éticos de una moralidad racional, es decir, de una educación moral que fundamenta la vida moral en principios racionales.

Así pues, su objetivo, el logro de una moral de principios, buscará la autonomía del sujeto al posibilitar la crítica a las normas morales convencionales, pero al mismo tiempo, al tener estos principios un carácter racional, serán universales, con lo que se niega el relativismo de las morales de situación.

$\mathrm{Y}$ es precisamente en este terreno donde se desarrollan las investigaciones de Kohlberg, que pretenden demostrar la universalidad de la forma del juicio moral; los trabajos de este autor son lo suficientemente conocidos como para que nos detengamos a exponerlos con detalle, pero sí pueden darse las líneas directrices de su teoría, que se pueden resumir en los cuatro puntos siguientes:

1) Establecimiento de 3 niveles de desarrollo moral, cada uno de ellos con dos estadios, haciendo así un total de 6 estadios, que pueden ser entendidos como estructuras formales de razonamiento moral.

2) Universalidad de estos estadios en las diferentes culturas y tradiciones morales.

3) Idea de que los estadios forman una secuencia invariante, lo cual significa dos cosas:

- En condiciones normales, el cambio de estadio ocurre hacia adelante, sin retrocesos.

- No puede haber saltos de estadio: los sujetos habrán de pasar de uno a otro sucesivamente.

4) Al ser modos o estructuras formales de razonamiento, cada estadio tendrá una manera propia de estructurar las obligaciones y los derechos morales, y cada uno de ellos subsumirá jerárquicamente a los anteriores, de modo que, a medida que el individuo asciende de estadio, puede hablarse de progreso moral, adquiriendo las 
propiedades de universalidad, prescriptividad, reversibilidad y generalidad, características de los principios morales.

Dicho en otras palabras y citando al propio Kohlberg en la presentación de sus teorías,

«Este enfoque recibe el nombre de cognitivo porque reconoce que la educación moral, como la intelectual, tiene sus bases en la estimulación del pensamiento activo del niño sobre cuestiones y decisiones morales. Y se le llama evolutivo porque entiende los fines de la educación moral como un movimiento a través de los estadios morales» ${ }^{1}$.

Por ello, y a pesar de que admite que la madurez del juicio moral es necesaria pero no suficiente para la acción moral, que es en definitiva el objetivo último de todo desarrollo en este ámbito, y aun admitiendo que no siempre un razonamiento moral se convierte en acción moral y que son necesarios otros factores como los motivos y las emociones del sujeto ${ }^{2}$, Kohlberg justifica así el que su enfoque se centre exclusivamente en el razonamiento moral:

- Dice, por una parte, que el juicio moral, pese a ser sólo un aspecto de la conducta y de la acción moral, es el de mayor importancia y mayor influencia en ella.

- Además, de entre todos los factores que influyen en la conducta moral, es el único exclusivo y distintivo de ella.

- Por último, mientras que la conducta moral es reversible, situacional o «perdible» en nuevas situaciones, el cambio de estadio en el juicio moral es irreversible.

Así pues, el presupuesto esencial de Kohlberg es subrayar la complejidad de la educación moral y la importancia del juicio moral para el desarrollo en este campo. Lo importante no es, como en el modelo convencional, la socialización e internalización de valores, ni como en el de la clarificación su exposición, sino la estimulación del desarrollo natural hacia estadios postconvencionales.

Pero precisamente su excesivo formalismo es una de las críticas más contundentes que se le han hecho a Kohlberg, acusándolo de reducir la educación moral a un asunto únicamente de pensamiento racional, considerado como mucho más importante que otros elementos intervinientes en el acto moral, como pueden ser los aspectos emocionales y afectivos.

Este objetivo prioritario del cultivo del juicio moral ha supuesto también un dejar al margen el contenido de la educación moral, al que considera como una instrucción adoctrinante que puede llegar a poner trabas al desarrollo de una forma autónoma, es decir, reflexiva y crítica, del juicio moral.

Resumiendo todo lo anterior en pocas palabras, puede decirse que la idea kohlbergiana de la educación moral tiene como objetivo el desarrollo de las capacidades formales del sujeto para el juicio moral, eludiendo la enseñanza de los contenidos concretos con el fin de no caer en la instrucción adoctrinante, opuesta a toda idea de autonomía, y eludiendo los aspectos motivacionales de la moralidad.

1. L. KOHLBERG, «El enfoque cognitivo-evolutivo de la educación moral», en JoRDAN; SANTOLARIA (eds.): La educación moral hoy. PPU. Barcelona, 1987, pp. 85-86.

2. L. KoHLBERG, op. cit., p. 94. 


\section{LA FILOSOFÍA MORAL DE R. S. PETERS}

Como planteábamos al principio, una noción determinada de «la moral», es decir, una teoría ética, es una condición previa esencial para todo enfoque serio de la educación moral.

Así pues, antes de empezar a hablar de los planteamientos de Peters sobre este tema, parece necesario exponer el concepto que este autor tiene de la moral, y esta necesidad es puesta de manifiesto por él mismo cuando dice:

«Toda explicación psicológica del desarrollo moral o de la educación moral deberá hacerse desde un punto de vista ético particular, porque la delimitación de la moral no es una cuestión neutral. Los psicólogos que trabajan dentro de marcos de aprendizaje piagetianos, freudianos o sociales tenderán a adoptar una posición ética implícita» ${ }^{3}$.

De acuerdo con esto, cada teoría ética se ocupará de un aspecto diferente del desarrollo y del aprendizaje moral. En la teoría de Peters, sus presupuestos se refieren a una MORAL RACIONAL, RELACIONADA CON REGLAS INTERPERSONALES. Con ello, el autor propone una postura intermedia entre el subjetivismo puro y la tradición, estrechamente conectada con el uso de la razón, la cual permitirá la adopción de una postura crítica por parte de los individuos hacia lo establecido, revisando el código en el que han sido educados y constituyéndose así en seres morales autónomos, sin por ello caer en el subjetivismo, al presuponer la comprensión de unos principios, surgidos, según Peters, como consecuencia de un choque de códigos diferentes, ante lo cual los hombres debieron meditar sobre estas cuestiones, determinando un código básico.

En su obra Desarrollo moral y educación moral Peters cita los principios siguientes:

* El primero de los que habla, la RACIONALIDAD, más que un principio propiamente dicho es la base de la concepción ética propuesta, en el sentido de que los principios no proporcionan una orientación concreta a las personas sobre lo que deben hacer, es decir, no contestan a la pregunta «¿Qué debo hacer ahora?», sino que la teoría moral basada en principios responde a esta otra: «En general, ¿cuáles son las cosas que pueden hacerse con fundamento en la razón?». De acuerdo con esto, los principios no funcionan como folletos de instrucciones, sino como guías para la acción; al ser de naturaleza no arbitraria y procedimental, lo que hacen es establecer las directrices generales acerca de los procedimientos a seguir para determinar lo que ha de hacerse.

* Otro principio es la IMPARCIALIDAD, que en el esquema de Peters resulta ser fundamental, entendiéndose por imparcialidad «la exigencia que excluye la arbitrariedad, la que sostiene que sólo se harán distinciones cuando existan diferencias pertinentes» ${ }^{4}$.

* La imparcialidad necesita, sin embargo, de otro principio complementario: la CONSIDERACIÓN DE LOS INTERESES DE LOS DEMÁS, que se concreta en actitu-

3. R. S. PETERS: Desarrollo moral y educación moral, ECE, México, 1984, p. 181.

4. R. S. PETERS: op. cit., p. 88. 
des como la benevolencia o la amabilidad, o en definitiva, en el sentimiento de sympatheia.

* Otro principio que le sirve de apoyo es la LIBERTAD, que para Peters consiste en que «en igualdad de otras circunstancias, debe permitirse que las personas hagan lo que quieran, es decir, deben ofrecerse razones para limitar a las personas en su búsqueda de lo que consideran bueno» ${ }^{5}$.

* Este principio de la libertad se relaciona muy estrechamente con la AuTONOMíA o desarrollo autónomo del individuo, destacando en ella la consideración que Peters hace de la tradición, en el sentido de que la autonomía implica tanto la independencia e integridad individual como la asunción de las obligaciones y reglas de la sociedad a la que se pertenece.

* El último de los principios referidos en esta obra es la TOLERANCIA, que puede tomar dos concreciones: EL RESPETO POR LAS PERSONAS y la disposición positiva hacia el PluRAliSMo, de modo que se posibilite la convivencia de las diferentes personas con distintas posturas individuales en base a estas convicciones morales compartidas que son los principios.

* A todos estos, en Ethics and Education, Peters añade los de la IGUALDAD y la VERACIDAD, vinculados, respectivamente, con la imparcialidad y con la consideración de los intereses de los demás ${ }^{6}$.

En este sistema de moral racional se presuponen dos cosas, esenciales en la teoría de Peters, y que, como veremos, constituyen el eje fundamental de sus críticas a Kohlberg:

1) El primer presupuesto es que, para que la capacidad racional se ejercite con eficacia, deberá estar apoyada por un grupo de pasiones racionales, que conforman el elemento afectivo o emocional de la moralidad, considerado tan importante como el cognoscitivo.

2) El segundo presupuesto es que, si, en una moral racional, los principios son procedimentales, es decir, no establecen en detalle lo que deba hacerse, limitándose a aportar una forma al juicio moral, estos principios generales han de concretarse en unas «normas básicas» que provean a la vida moral de un contenido detallado.

\section{INSUFICIENCIA DEL PLANTEAMIENTO DE KOHLBERG: LA PROPUESTA DE PETERS EN EDUCACIÓN MORAL TEMPRANA}

Una vez examinados los presupuestos filosófico-morales de Peters, podremos entrar a considerar su propuesta de educación moral, que, para él ha de centrarse, como para Kohlberg, en los principios éticos de una moralidad racional.

Pero es aquí donde ambos autores muestran planteamientos diferentes:

* Mientras para Kohlberg el modelo pedagógico ha de poner el acento exclusivamente en la consideración de la educación moral como un asunto de mero desarrollo del razonamiento y el espíritu crítico, donde lo que se pretenda sea el

5. R. S. PETERS, op. cit., p. 90.

6. R. S. Peters, Ethics and Education. Scott Foresman. Chicaco, 1968. Peters trata de la igualdad en las páginas 117 y ss., y de la veracidad en la p. 172. 
desarrollo de la forma del pensamiento y el juicio moral del sujeto para lograr un agente autónomo (es decir, perteneciente a los estadios superiores), para Peters, al lado del juicio moral han de situarse los elementos emocionales y afectivos, que también están presentes en toda acción moral.

* Por otra parte, esta importancia que da al desarrollo de la forma del juicio moral ha supuesto el que Kohlberg dejara a un lado el contenido de la educación moral, a causa de que, para él, enseñar un contenido equivale a transmitir un código y con ello se puede incurrir en una instrucción adoctrinante que dificulte el desarrollo de una forma crítica y reflexiva - autónoma- del juicio moral.

A causa de esto, Kohlberg ha restado valor a la tarea educativa de fomentar la adquisición de los hábitos, considerando que el hábito, por definición, supone un comportamiento irreflexivo e inconsciente del sujeto, opuesto a la conducta autónoma que él pretende como objetivo del desarrollo de una forma racional del juicio moral.

Así pues, y a pesar de que entre Peters y Kohlberg hay algunos otros puntos de discrepancia, son éstos los que el propio Peters considera como más relevantes a la hora de elaborar o plantear una propuesta de educación moral, que es lo que pretendemos analizar aquí, por lo que es a ellos a los que vamos a dedicar nuestra atención preferente, desarrollando el planteamiento de otras «críticas menores» en función del esquema derivado de éstas.

Sin embargo, antes de entrar en este análisis, puede decirse que la diferencia de los planteamientos de Kohlberg y Peters encuentra un precedente en la Grecia Clásica en las posturas del Sócrates platónico y de Aristóteles, con cuya exposición, que no pretende ser exhaustiva, podrá apreciarse cómo el tema de que tratamos ya había sido objeto de interés en los comienzos del pensamiento moral.

Efectivamente, aun en medio de la duda socrática acerca de si es posible enseñar la virtud, puede verse la idea de que la moralidad consiste en alguna forma de conocimiento y la inmoralidad en alguna forma de ignorancia, como se aprecia en el siguiente texto del Protágoras de Platón:

«Sentado esto, sostengo que no hay cosa más ridícula que decir, como vosotros hacéis, que un hombre conociendo el mal como mal y estando en su voluntad no entregarse a él, se entregue sin embargo, porque se ve arrastrado por las pasiones; y que un hombre, conociendo el bien, rehúse practicarlo a causa de algún placer presente que le aleje de él».

Pese a esto, la «ciencia» de la moralidad no es sólo un mero conocimiento, sino también un modo de razonamiento moral: para actuar de un modo ético no se necesita ser sabio sino prudente, es decir, obrar tras una deliberación que mida las posibles consecuencias de la acción. También en el diálogo Protágoras se dice:

«...No es sólo por falta de ciencia, sino la falta de esa ciencia especial que enseña a medir. $Y$ toda acción en la que puede haber engaño por falta de ciencia, ya sabéis que es por ignorancia. Por consiguiente, el ser vencido por el placer es el colmo de la ignorancia».

Este intelectualismo socrático suscita las críticas del pragmatismo aristotélico, cuando dice: 
- «Sócrates solía investigar qué es la virtud, pero no de qué manera se produce». (Ética Eudemia).

- «Tampoco tratándose de la virtud basta con conocerla, sino que se ha de procurar tenerla y practicarla (...). Ciertamente, si los razonamientos bastasen para hacer buenos a los hombres reportarían justamente muy grandes remuneraciones, como dice Teognis, y sería preciso procurárselos; pero (...) resultan incapaces de excitar a la bondad y a la nobleza al vulgo». (Ética a Nicómaco).

Así pues, en Aristóteles se pone de manifiesto el componente afectivo de la moralidad: resalta la inutilidad del conocimiento por sí solo para lograr una acción moral, evidenciando la fuerza del elemento pasional, sentimental, y de ahí la importancia que concede al hábito, cuando dice en la Ética a Nicómaco que:

«Las virtudes no nacen en nosotros ni por naturaleza ni en oposición a ella, sino que, siendo capaces de ellas, las iniciamos y perfeccionamos mediante la praxis».

Estas referencias históricas no difieren mucho de los planteamientos de Kohlberg y Peters, como lo especifica este último cuando le formula sus críticas al formalismo del primero, aunque sobre esto hay que decir que, en líneas generales, Peters acepta la validez de las teorías de Kohlberg sobre el desarrollo moral, advirtiendo que su intención no es tanto criticar como complementar el enfoque allí donde éste resulta insuficiente, como se comprueba cuando dice:

«Éstas, sin embargo, son críticas cuya nota común más decisiva es la exigencia de que algunas de las afirmaciones más importantes de la exposición de Kohlberg deberían ser formuladas más detalladamente» ${ }^{7}$.

Así pues, la primera de las críticas que Peters le hace a Kohlberg es que su sistema no se ocupa de los ASPECTOS AFECTIVOS DEL DESARROLLO MORAL. La importancia que da este autor a la moralidad regida por principios y a los factores cognoscitivos es de sobra conocida, pero con su planteamiento se corre el riesgo de que un lector de la calle piense que éstos son los principales ingredientes del desarrollo moral, mientras que, como Kohlberg mismo admitiría, su teoría tan sólo se aplica a una parte del proceso evolutivo en el ámbito de la moral.

Si el objetivo del desarrollo y de la educación moral es la acción moral, el mero desarrollo del razonamiento moral es importante pero insuficiente, adquiriendo importancia los elementos más olvidados por el modelo kohlbergiano, como son los factores afectivos, puesto que para que la acción sea consistente con el juicio se requiere no sólo la corrección de éste, sino, además, un cierto «apasionamiento» o sentimiento, siempre y cuando éste no pierda su carácter racional. Por consiguiente, la educación no debe centrarse solamente en el desarrollo del razonamiento moral sino que se deben crear y favorecer unas condiciones que contribuyan a que se ponga en acción lo propuesto por el razonamiento moral.

Para empezar a hablar del tema, hemos de acudir a la descripción filosófica que Peters hace de la pasión en su trabajo «La Razón y la Pasión». Según él, la pasión, que en lenguaje ordinario significa un sentimiento exaltado que domina al indivi-

7. R. S. PeTERs, «La educación y el desarrollo humano», en DEARDEN, Educación y desarrollo de la razón. Narcea, Madrid, 1982, p. 473.

8. R. S. PETERS, «La razón y la pasión», en DeARDEN, Educación y desarrollo de la razón, op. cit., pp. 201-219. 
duo, en sentido filosófico puede ser definida como «algo que proporciona un estímulo para actuar», y ese algo no tiene por qué ser ni irracional ni no razonable.

- Por «irracional» Peters entiende un comportamiento que «se desvía del modelo de comportamiento que persigue un fin», y que se desvía, además, de una manera consciente, es decir, con conocimiento de ese modelo y de la propia desviación de él.

- Por «no razonable» entiende el comportamiento de una persona que, teniendo razones para actuar de una manera, no las toma en consideración, prestando mayor atención a otras razones de menos peso para actuar de otro modo.

De acuerdo con esto, las pasiones o los sentimientos no tienen por qué ser necesariamente irracionales ni no razonables, aunque pueden serlo, si las valoraciones relacionadas con ellas se hacen de un modo intuitivo o poco reflexivo (en el caso de las pasiones irracionales) o si se hacen de una manera inadecuada (en el caso de las no razonables).

De este modo las pasiones, los aspectos afectivos de la moral, están íntimamente relacionadas con el uso de la razón, ya que el ejercicio de la moralidad es inexplicable sin ellos. Por eso, mientras Kohlberg efectúa una oposición tajante entre razón y sentimiento o emoción, en Peters no se da esta ruptura: la propia razón de los conceptos morales que se asumen constituye una respuesta afectiva a otras personas en la sociedad, que es definida como sentido social o altruismo.

Por lo tanto, al lado del principio de la Justicia, que para Kohlberg es el «principio de los principios», Peters propone otros principios fundamentales, que figuran entre los que ya se han analizado antes, como el de la IMPARCIALI$\mathrm{DAD}^{9}$, sin el cual el individuo no podría efectuar consideraciones distintas de su propio punto de vista y como, sobre todo, el de la CONSIDERACIÓN DE LOS INTERESES DE LAS PERSONAS ${ }^{10}$, que se concreta en la «benevolencia», y que para él es tan importante en la moral como el principio de la justicia, pese a que ni Piaget ni Kohlberg traten de él, debido, quizá, a su ascendencia kantiana.

La concesión de una gran relevancia a este principio lleva a Peters a plantearse sus implicaciones en el tema de la educación moral, analizando los estudios acerca de su desarrollo. Para ello, cita a Martin Hoffman ${ }^{11}$, quien elabora una teoría del desarrollo del altruismo, en la que el punto de partida es el supuesto de que en el hombre coexisten innatamente la motivación egoísta y la altruista, por lo que, de lo que se trata en educación moral, es de explicar cómo se puede desarrollar la motivación altruista en el individuo en base a la capacidad humana de asunción de roles, es decir, de experimentar el estado interior de otras personas que se encuentran en distinta situación; sin entrar en la polémica de si el sentimiento de empatía es innato o adquirido, controversia que se remonta ya a Hobbes, Hume, etc., Peters mantiene la existencia de varias etapas:

9. R. S. PETERS: op. cit., p. 206.

10. R. S. Peters: Desarrollo moral y educación moral, op. cit., p. 224.

11. HoFrMAN: «Empathy. Role-taking, Guilt and Development of Altruistic Motives», en T. LicKONA, (comp.): Moral Development and Behaviour. Holt, Rinehart and Winston. New York, 1976. Citado por Peters en op. cit., p. 227. 
1) La primera comienza cuando el niño es capaz de entender la permanencia de los objetos y las personas; aquí, aunque la perspectiva del niño siga siendo egocéntrica, puede ya sentir empatía por algo o alguien distinto de sí mismo.

2) El siguiente paso es cuando el egocentrismo es sustituido por el desempeño de papeles, y el niño empieza a advertir que los otros tienen diferente punto de vista que el suyo (7-8 años según Piaget, varios años antes, según Hoffman).

3) Esta etapa culmina cuando el niño advierte que otros individuos tienen su propia identidad personal y sus propios estados internos, reaccionando ante sus penas, y más adelante, comprendiendo ya no sólo las penas de los individuos sino las de todo un grupo (lo que Hume llamó el paso de la «benevolencia limitada» al «sentimiento por la humanidad»).

Para desarrollar la motivación altruista, Hoffman sugiere cuatro cosas: permitir que el niño tenga una dotación normal de experiencias desdichadas en lugar de protegerlo de ellas; proveerlo de oportunidades para la asunción de papeles y para ayudar a otros; alentarlo a que se imagine en el lugar de otros; exponerlo a modelos que se comporten de manera altruista.

Pese a reconocer lo sugerente del planteamiento especulativo de Hoffman, Peters lo califica de grosero, en el sentido de que la preocupación que un niño siente por alguien desconocido siempre será distinta de la que siente por alguien que conoce más, por lo que piensa que puede refinarse, y para ello hace referencia al trabajo de Secord y Peevers ${ }^{12}$ sobre la percepción que tienen los niños acerca de otros individuos. Al modelo emocional de Hoffman, estos autores le dan un núcleo cognoscitivo, al describir el altruismo siguiendo las etapas de Piaget:

- Así, hablan en primer lugar de una etapa egocéntrica, en la que los niños sienten simpatía, en mayor medida, hacia quienes los aman o los miman.

- Vendría después una etapa de realismo, que es una etapa de la mutualidad, de consideración de los amigos y lealtad hacia ellos como miembros de un mismo grupo.

Y por último, Secord y Peevers hablan de una etapa final, en la que la reacción simpática hacia otra persona que sufre se dirigirá hacia ella como un individuo, adoptando una actitud objetiva, de preocupación desinteresada, por otro ser humano.

Esta explicación, a pesar de que no es más que una historia probable apoyada por un número limitado de estudios psicológicos, tiene para Peters el valor de ser consistente con la visión del desarrollo cognoscitivo de Piaget y Kohlberg, con lo que perfectamente puede servir para completar éste en su insuficiente tratamiento de los aspectos afectivos de la moralidad.

Pero, además de las motivaciones positivas que acabamos de citar (simpatía y consideración de los demás), el aspecto afectivo de la moralidad también incluye motivaciones negativas, como la vergüenza y la culpa.

* La primera de ellas, la VeRgüENZA, es la más clara de estas motivaciones, y puede ser definida como el sentimiento de un individuo al tomar conciencia de que no ha estado a la altura de lo que los demás esperaban de él, o de que no ha ejerci-

12. P. F. Secord y B. H. Peevers: «The Development of Person Concepts», en T. Mischel (comp): Understanding other Persons. Blackwell, Oxford, 1974. Citado por Peters en op. cit., p. 227. 
tado las virtudes estimuladas en su plan de vida. En la vergüenza intervienen como agentes principales otras personas, aunque se conecta con el respeto por uno mismo, al suponer un sentimiento de disminución del yo o de autodenigración. En su gráfica «Motivos para la acción moral» (From Is to Ougth), Kohlberg no se refiere a la verguienza, pero completando el esquema kohlbergiano, Peters la incluye en las etapas 3 y 4 .

* La Culpa resulta más difícil de tratar para este autor, debido a la influencia de las teorías psicoanalíticas, que la describen como un sentimiento debido a la violación de las reglas entendidas como algo objetivo, asociándose con la ansiedad y el temor al castigo, es decir, que, explicándola en función del Superego, la definen solamente como una «culpa de autoridad», con lo que, en el esquema de Piaget y Kohlberg, sólo podría situarse como motivación negativa para la acción moral en los estadios de realismo. En su intento de completar este esquema, Peters amplía este concepto, hablando de otro tipo de culpa, que se experimenta cuando se lleva a cabo una acción contraria a nuestro sentido de lo correcto y lo justo, propia, por lo tanto, de las etapas más avanzadas de desarrollo moral.

Así, mientras la vergüenza era un sentimiento de pérdida de autoestima, la culpa puede ser descrita como el aspecto negativo del altruismo que ha sido analizado en la hipótesis de Hoffman, al ser experimentada tras la violación de los derechos de los demás y tras las lesiones que esto les causa, al no respetar el principio de la justicia de Kohlberg.

La otra gran crítica que Peters le plantea al esquema kohlbergiano es la INADECUACIÓN DE SU TRATAMIENTO DEL TEMA DEL CONTENIDO en la educación moral, y será a la que nos dediquemos con más detalle, por llevar implícitas ciertas críticas menores al propio sistema.

Antes de entrar en el análisis de la crítica, se hace conveniente examinar la idea que Peters tiene de este término dentro de la moral postulada por él, que, como ya vimos, es una moral basada en principios racionales. Y, como sostiene en su obra Desarrollo moral y educación moral, la comprensión de lo que significa el uso de la razón en la moral requiere una distinción previa entre FORMA y CONTENIDO.

Para explicarlo, pone como modelo las creencias acerca de la forma de la tierra: un contenido al respecto podría ser, por ejemplo, que la tierra es redonda. Pero este contenido único podría sostenerse de distintas formas: podría creerse por haberse leído en un libro, o a través del examen de pruebas científicas, etc. Utilizando otro ejemplo, esta vez de la esfera moral, alguien podría creer que el juego de azar es malo porque siempre le han dicho que lo era, o bien podría haber llegado a esta conclusión a través de la reflexión sobre esta práctica y de la constatación del sufrimiento que produce a las personas.

Como describe Peters en «La educación y el desarrollo humano», en el sistema kohlbergiano se establece una diferencia entre el contenido de las creencias morales, que depende de la cultura en la que éstas se produzcan, y la forma de estas creencias, que depende del grado de desarrollo moral de los individuos, y que es una invariante cultural.

Por ejemplo, para Kohlberg, en el dilema de Heinz (dilema utilizado por él en sus diferentes estudios para identificar el estadio moral de un sujeto, que plantea la 
cuestión de un hombre que roba una medicina para salvar a su mujer moribunda, tras intentar conseguir el dinero para comprarla y pedir al farmacéutico que le baje

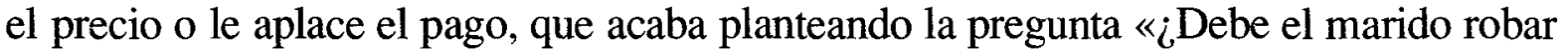
el medicamento o no debe hacerlo?»), el contenido del juicio moral es la decisión que adopte el sujeto, su respuesta a esta pregunta, mientras que la forma de su juicio moral vendrá dada por las razones que exponga para justificar la respuesta dada.

Pese a esta distinción, en el esquema no se le presta la suficiente atención al tema del contenido, y esta omisión no es, según Peters, involuntaria en Kohlberg, sino que, para él, éste es un tema secundario, cuando afirma que un «saco de virtudes» carece de importancia en el equipo moral de una persona.

En contra de esta opinión, Peters sostiene la importancia del contenido en moral y en educación moral, haciendo las siguientes observaciones a Kohlberg:

- En primer lugar, hace notar que los propios estadios 5 y 6 kolbergianos están repletos de ese «saco de virtudes» que él mismo rechaza, como pueden ser la justicia, la benevolencia, el respeto y la veracidad que componen el nivel de la moral regida por principios de Kohlberg.

- Pero además, Peters da otro tipo de razones para recomendar que se preste más atención al contenido como aspecto de la moralidad:

- La primera de ellas parte de la consideración del carácter social de la moral, que, como veíamos antes, influye en la prioridad que da este autor a ciertos principios, como la consideración de los intereses de la gente; así, argumenta que, puesto que si tanto los niños como los adultos pertenecientes a los estadios convencionales tienen que vivir con los demás, es esencial que posean unas cuantas virtudes en su «saco»; como dice en Desarrollo moral y educación moral.

«Hobbes observó un aspecto humilde de la condición humana: el hecho de que un hombre puede ser muerto por un niño cuando se encuentra dormido. Son también innumerables los riesgos que corren los niños pequeños en la sociedad industrial moderna. Así pues, por razones de seguridad social y de autoconservación, debe enseñarse a los niños pequeños un código básico que, una vez interiorizado, regule su comportamiento hasta cierto punto cuando no estén siendo vigilados. Debemos tomar en cuenta también el hecho de que gran número de personas no alcanzan el nivel de moral racional. Por lo tanto, por razones sociales obvias, si la moral de tales personas ha de ser irreflexiva, su contenido tendrá una importancia decisiva» ${ }^{13}$.

- Pero es que, además, como señala en su trabajo «Ambigüedades en la educación liberal y el problema de su contenido» ${ }^{14}$, si la autonomía no es más que una cuestión de grado, una actitud mental y no una condición lograda de una vez por todas, en la esfera moral, la persona autónoma actúa muchas veces de acuerdo con una serie de reglas sobre las cuales no ha reflexionado; dicho de otro modo, el individuo autónomo no es alguien que opere siempre al nivel de una moral regida por principios, sino que, además, funciona en gran medida por las recompensas y castigos, por obediencia al mandato de la autoridad, etc. Pese a esto, y contrariamente al que no ha al-

13. R. S. PETERS: Desarrollo moral y educación moral, op. cit., p. 205.

14. Incluido en STRIKE-EGAN: Ética y política educativa, Narcea, Madrid, 1981, pp. 17-32. 
canzado la autonomía moral, el individuo autónomo es capaz de hacer esas mismas cosas porque considera que emanan de sus principios fundamentales, y puede actuar de modo diferente, cuestionándose ciertas normas establecidas sobre la base de sus propias convicciones, es decir, con una actitud racional ante la autoridad y la tradición, situándose de este modo en el tercer nivel de Kohlberg. No obstante, y como venimos diciendo, una gran parte del comportamiento de los individuos moralmente autónomos está regida por la moral del segundo nivel: sin meditar sobre los principios implicados en sus acciones, se abstienen de robar, pagan lo que deben o respetan el orden de las colas, con lo que se puede comprobar que incluso en las personas autónomas, la etapa convencional de la moralidad sigue teniendo una gran importancia.

- El segundo tipo de razones que alega Peters para prestar atención al contenido de la educación moral es de carácter individual: aunque se dejara de lado la cuestión del comportamiento social, existirían otros dos motivos para no despreciar el contenido: 1) el primero consiste en que aprender a respetar una regla, a través de la generalización a partir de unos contenidos concretos, es un requisito previo para la aplicación de las reglas de una forma autónoma, y ésto sólo lo pueden aprender los niños «generalizando su experiencia de captación de cierto 'saco de virtudes' particulares» ${ }^{15}$. 2) por otra parte, el ejercicio de una forma de moral regida por principios ha de hacer referencia a un contenido determinado que resulta ser necesario para poder operar moralmente. Como dice Peters, «resulta imposible la concepción de tal moral regida por principios que opera en un vacío, separada de un contenido determinado ${ }^{16}$. $\mathrm{O}$, dicho en otras palabras, el contenido es lo que, en definitiva, valida cualquier forma de juicio moral.

Por ello, y si los principios, como ya vimos, no establecen lo que deba hacerse, es decir, no dotan de contenidos a la moral, limitándose a aportar una forma de pensamiento que estructura la experiencia, una educación moral que, como en el esquema de Kohlberg, sólo se ocupe del desarrollo de la forma de la conciencia moral, resulta incompleta, y de ahí que Peters piense que hay que añadirle algo, puesto que no abarca todo el proceso de desarrollo moral sino sólo una parte; por ello, y sin rechazar la cuestión de la autonomía, que por supuesto es esencial y deseable como meta de toda educación moral, para alcanzar el más alto grado posible del desarrollo es esencial para este autor resaltar, como vimos que hace, la dimensión social de la moral, por lo que la propuesta de Peters se centra en tres puntos:

1. No se puede dar una descripción del desarrollo de una forma racional de la moral sin prestar al aprendizaje del contenido más atención de la que Kohlberg le presta.

2. El segundo punto de la propuesta de Peters es que, pese a que se dice no skinneriano, y en sus diferentes obras critica a Skinner y al conductismo en general $^{17}$, Peters mantiene que la descripción kohlbergiana del desarrollo de una forma

15. R. S. PETERs: Desarrollo moral y educación moral, op. cit., p. 206.

16. R. S. Peters: op. cit., p. 207.

17. Puede verse: «Survival of the Soul», en Psycology and Ethical Development y The Concept of Motivation. 
racional de la moral es compatible con un tipo skinneriano de descripción del aprendizaje del contenido en la primera infancia, como veremos más adelante, al analizar el tema de los refuerzos.

3. Por último, piensa también que han de buscarse y emplearse aquellas formas de aprendizaje del contenido que no impidan ni dificulten el desarrollo de una forma racional de la moral.

Estos son, en definitiva, los puntos de los que parte Peters para elaborar su teoría del contenido en desarrollo y educación moral que, como ya se ha dicho, lo que pretende es completar la propuesta de Kohlberg. Por ello, y mientras la consideración que éste hacía de los estadios morales tenía únicamente un carácter lógico y epistemológico, podemos hablar de que en Peters se introduce el elemento de lo psicológico en el desarrollo y el aprendizaje moral, como ya hemos insinuado cuando tratábamos el tema de los aspectos positivos de la moralidad, y como puede verse a la hora de tratar el tema de los contenidos y de elaborar un currículum de educación moral, es decir, a la hora de determinar qué contenidos deben enseñarse y cómo ha de hacerse.

Para explicarlo, Peters ${ }^{18}$ describe las etapas de una forma racional de la moral en la teoría de Piaget y Kohlberg:

0) Alude a una etapa previa, prerracional e instintiva, para pasar después a describir.

1) Una primera etapa, la etapa EGOCÉNTRICA; en ella, el niño hace lo codificado para evitar el castigo u obtener recompensas. Esto quiere decir que, al contrario que en la etapa prerracional, ya existe en él un aparato cognoscitivo y afectivo básico, y ya percibe una cierta conexión entre los medios y los fines; es decir, ya posee una forma primitiva del concepto categórico de «causalidad». Para lograr este desarrollo, el niño ha de tener oportunidades de manipulación de las cosas, ya que este marco de conceptos no puede impartirse, como ya había afirmado Piaget, mediante una enseñanza específica, sino que es necesario que se le proporcionen abundantes experiencias concretas mediante la cuales pueda adquirirlos, estimulándole adecuadamente. Esto explica la objeción que hace Kohlberg a una enseñanza específica, e introduce el concepto de «estimulación cognoscitiva» entendida como la provisión de condiciones en las que el niño pueda empezar a desarrollar sus capacidades racionales, sobre el que más adelante volveremos. Pero tampoco debe minimizarse, según Peters, el papel de la instrucción en el desarrollo de esta etapa de la moralidad, dejando que los niños lo descubran todo por sí mismos sin la provisión de un ambiente social estimulante y estructurado por parte del adulto.

2) En segundo lugar, Peters describe la etapa TRASCENDENTE; esta etapa, de realismo moral, es decisiva en el desarrollo del niño, porque encierra el entendimiento de lo que significa respetar una regla y aceptarla como algo que limita nuestra conducta; así pues, la conformidad con las reglas ya no es entendida como un procedimiento para evitar el castigo y obtener recompensas, sino que, aun sin tener todavía ninguna noción de la validez de las reglas, los niños las aprecian como algo cuya existencia depende del grupo de compañeros y de la autoridad. La identificación y

18. R. S. PETERS: Desarrollo moral y educación moral, op. cit., pp. 191 y ss. 
la imitación son, pues, muy importantes para el aprendizaje en este período, introduciéndose el ejemplo como recurso educativo, además de que en esta etapa de conformidad con las reglas es muy importante para el desarrollo moral, puesto que, si es cierto lo que dicen Piaget y Kohlberg, todos los individuos han de pasar por ella para alcanzar la etapa de la autonomía, y puesto que, por otra parte, no siempre será superada, sino que muchos individuos se quedarán en ella si no disfrutan de la suficiente estimulación y si se utilizan con ellos determinadas técnicas que imposibiliten la evolución a la siguiente etapa, que sería la

3) Etapa Autónoma. Podemos hablar de tres aspectos en la autonomía: el primero es el aspecto de la autenticidad; esta noción sugiere que la persona acepta ciertas reglas por sí misma, y ya no por la aprobación o desaprobación de sus compañeros o de las autoridades. El segundo es el aspecto de la autonomía como capacidad para reflexionar sobre las reglas y someterlas a la crítica desde el punto de vista de su validez y aplicabilidad, tomando la perspectiva de los otros. El tercero es el aspecto de la autonomía como fuerza de voluntad, o capacidad para mantener un juicio o una conducta frente a las inclinaciones contrarias.

Pero así como decíamos que hay técnicas o influencias sociales (como por ejemplo la adoctrinación) que desalientan a los individuos para que no vayan más allá de la segunda etapa de la moral, puede haber otras que, por el contrario, estimulen su desarrollo hacia este período autónomo. En concreto, estos tres aspectos de la autonomía a que nos acabamos de referir, no se desarrollan por sí solos en los jóvenes, sino que los adultos, el sistema social y educativo, han de proporcionarles ocasiones adecuadas para que lo hagan:

* La autenticidad está muy ligada a la capacidad de experimentar preocupación por los demás: no hacer algo sólo por evitar el castigo, sino por lo que puede significar de daño para otras personas; aunque en un primer momento esto no funcione como un principio, los niños pueden ya sentirlo, y con el desarrollo se puede convertir en principio, si previamente sus relaciones sociales le predisponen para ello.

* El desarrollo del razonamiento tiene vinculaciones con la reflexión sobre las reglas, la cual, además de por «estimulación cognoscitiva» puede lograrse a través de las técnicas llamadas «de inducción», entre las que pueden citarse la explicación o el señalamiento de las consecuencias de la acción, empleadas mientras el niño no comprende las razones.

* En cuanto al tercer aspecto de la autonomía, la fuerza de voluntad, tiene también para Peters una gran importancia, a pesar de que comúnmente se haya asociado a morales tradicionales y se haya descuidado. En ella tienen relevancia los hábitos, de los que más tarde se hablará, pues se trata de ejercitar la voluntad frente a las inclinaciones contrarias. El modo de desarrollarla es sensibilizando al niño hacia las consideraciones positivas que apoyan de una forma permanente la resistencia a estas inclinaciones contrarias.

Lo cierto es que, a pesar de que Kohlberg no deja de justificar su intelectualismo a la hora de atender a la relación entre juicio moral y acción moral en base a la idea de que cuanto más alto es el nivel de razonamiento moral alcanzado por una persona hay una mayor propensión para actuar más coherentemente con ese juicio, aportando incluso datos empíricos para demostrarlo, parece aceptar y asumir la crí- 
tica que Peters y otros autores le hacen de que su sistema se ocupa de modo insuficiente del tema del contenido y que concede menos importancia de la que debiera a la etapa convencional de la moralidad, hasta el punto de que, en las reformas parciales que va introduciendo en su paradigma, desde el año 1979, ante la Asociación Americana de Psicología, admite que su postura «no es meramente socrática y desarrollista, sino también 'adoctrinante', buscando formar un buen miembro de la comunidad, un buen ciudadano» ${ }^{19}$.

Como conclusión de esta primera parte del tema del contenido, en la que Peters trata de responder a la cuestión del «¿por qué preocuparse por él?», podemos citar un párrafo del libro Desarrollo moral y educación moral en el que se exponen de un modo resumido las razones que él da, y se plantea la problemática que de ello se deriva y que a continuación trataremos. El texto dice así:

«Por supuesto, la etapa de la moral convencional tiene algunos defectos. No estoy defendiendo su superioridad moral, sino sólo su necesidad lógica y práctica en cualquier explicación de la vida moral. Estoy insistiendo en que deberá reflexionarse más sobre su contenido y sobre los métodos de su enseñanza. En virtud de que los niños pequeños no pueden apreciar su justificación en términos de principios, y puesto que deben aprender tal contenido básico por las razones que he bosquejado, el problema consiste en el empleo de métodos de enseñanza del código a los niños pequeños, métodos que sean eficaces sin ser adoctrinantes y que allanen el camino hacia una moral posterior, regida por principios $»^{20}$.

Una vez analizadas las razones que alega Peters por las que considera importante prestar una mayor atención al contenido de la moral, y una vez aceptada la condición de indispensable de una cierta clase de contenido en la educación moral, surge como consecuencia un segundo interrogante: «¿QUÉ TIPO DE CONTENIDOS DEBEN ENSEÑARSE EN EDUCACIÓN MORAL?».

Esta es una pregunta relevante, ya que, en todo caso, el niño siempre recibe un contenido: aun cuando los adultos no se lo transmitan deliberadamente, siempre introducirán a los niños en el tipo de contenidos que a ellos les parece importante, puesto que el mundo en el que niño debe habitar está en gran medida ya estructurado por los papeles, las reglas, las actividades y las relaciones de los adultos. Por eso, cualesquiera que sean las técnicas de educación moral que se usen de una manera explícita, el niño siempre estará expuesto de un modo implícito a un cierto contenido.

De este modo, Peters introduce el elemento teleológico de la educación: decir que siempre se transmite un contenido es lo mismo que decir que siempre se educa para obtener un determinado tipo de educandos; los contenidos en educación moral se convierten, pues, en fines educativos, y de ahí la importancia que adquiere para este autor la pregunta por el «qué tipo de contenidos se deben enseñar». Para contestarla, Peters ha de completar sus planteamientos de filosofía moral, poniendo, al lado de los «principios de procedimiento» que ya se analizaron antes, un conjunto de «normas básicas» de la vida social, que no son supuestos del discurso práctico como los principios fundamentales, sino que se refieren a unos hechos empíricos,

19. L. KoHlberg: «Moral Education for a Society in Moral Transition», en Muson: Education Leadership (1979), 33, p. 57.

20. R. S. PETERS: Desarrollo moral y educación moral, op. cit., p. 236. 
relativos a la situación del hombre sobre la tierra, concretando los principios. De este modo, como el autor las describe en «Reason and Habit: The Paradox of Moral Education $\gg^{21}$ las normas básicas son, de alguna manera, universales, «tan importantes para cualquiera que viva en sociedad que pueden considerarse casi como definiciones de una sociedad», por lo que «las normas sociales serían las que se pueden justificar bajo cualesquiera situaciones sociales». Sin embargo, a la hora de determinar cuáles son para Peters esas normas básicas, nos encontramos con que no se hallan especificadas de un modo concreto en ninguno de sus escritos, sino que están dispersas por todos ellos, presentadas en diferentes combinaciones. En su estudio sobre la teoría de Peters, Royce ${ }^{22}$ mantiene haber encontrado referencia a 9 normas distintas, pero no garantiza ofrecer el esquema de Peters al completo. Estas normas son las siguientes: la no lesión, mantener los compromisos, el cuidado del joven y la propiedad, la regulación de la reproducción, el asesinato ${ }^{23}$, la puntualidad, la honradez $^{24}$, y a veces la veracidad y el robo. En esta lista, a veces las normas se presentan en combinaciones variables de dos o tres, seguidas por un «etc.», por lo que no puede saberse si la relación está ya completa o si habría que añadir alguna más.

De acuerdo con esto, y aceptando la estructura de la moralidad en base a unos «principios de procedimiento» y a unas «normas básicas», estructura de la que quedan excluidas las «reglas controversiales, tales como las referentes a las prácticas sexuales y a los sindicatos ${ }^{25}$, Peters responde así a la pregunta por cuál ha de ser el contenido de la educación moral: ha de ser tanto lo referido a lo que en la etapa autónoma serán los principios éticos como a las normas básicas que son sus concreciones:

«Estas virtudes serán, o bien las que se refieren a la preocupación por los demás, a la veracidad y a la justicia, que posteriormente funcionarán como principios fundamentales, o bien serán normas como no robar y cumplir las promesas, que se pueden defender por referencia al principio fundamental de que son indispensables para la vida social en cualquier condición concebible. Si, debido a la influencia del aprendizaje temprano, los niños desarrollan una actitud más bien inflexible ante estas reglas, al menos la tendrán para reglas que probablemente serán apropiadas para cualquier forma de vida social que encuentren en una época de cambio social» ${ }^{26}$.

La cuestión que sin embargo más interesa a Peters en su tratamiento del contenido es la de «¿CÓMO DEBERÁ ENSEÑARSE ÉSTE?». Para abordar este tema ha de determinarse previamente cuál es el concepto petersiano de enseñanza. Al respecto, el autor hace alusión a los estudios de Piaget y Kohlberg sobre el desarrollo de una forma racional de la moralidad, diciendo que, para Kohlberg, la enseñanza del contenido es algo totalmente distinto al desarrollo de la forma de la moralidad,

21. R. S. Peters: «Reason and Habit: The Paradox of Moral Education», en NiBLet (ed.): Moral Education in a Changing Society, Faber and Faber, London, 1963. Traducción castellana en Desarrollo moral y educación moral, op. cit., pp. 54-74.

22. RoYCE: «R. S. Peters and Moral Education, 2: Moral Education in Practice». Journal of Moral Education, vol. 13, n. 1 (enero, 1984) pp. 9-16.

23. En Ethics and Education, op. cit., pp. 174-175.

24. En Psycology and Ethical Development, Allen and Unwin, London, 1974, p. 297.

25. R. S. PeTERs: Desarrollo moral y educación moral, op. cit., p. 234.

26. R. S. PETERS: «Forma y contenido de la educación moral», en JoRdan; SANTOLARIA (eds.): $L a$ Educación moral hoy, op. cit., p. 130. 
que, como ya se apuntó más arriba, es lo que en definitiva ha de tratar de hacer la educación moral para este autor, y ello a través de la estimulación cognoscitiva: lo que no debe hacerse, según Kohlberg, es enseñar contenidos morales, pues se corre el riesgo de caer en el adoctrinamiento. Pero es aquí donde Peters le formula sus críticas a la, según él, estrecha visión kolbergiana de lo que significa enseñar, equiparándolo con adoctrinar, con decir algo explícitamente, en contraposición con la estimulación cognoscitiva, de la que puede servir como ejemplo Sócrates con el esclavo en el diálogo platónico Menón. Sin embargo, en su crítica, Peters mantiene que sin duda Sócrates estaba enseñando al esclavo aunque no le dijera nada de un modo explícito, porque «enseñar es conducir a que alguien aprenda algo indicándole de un modo u otro lo que debe ser aprendido con tal que esté a su nivel de entendimiento. Esto se puede hacer sin decir las cosas explícitamente $»^{27}$. De acuerdo con esto, la respuesta de Peters a la cuestión de «¿Cómo debe enseñarse el contenido?» es clara: «A ciencia cierta, de cualquier manera que contribuya a que aprendan esas reglas y que no embote su capacidad de desarrollar una actitud autónoma frente a ellas» ${ }^{28}$, es decir, haciendo referencia al carácter psicológico y no sólo epistemológico de las etapas de desarrollo moral a que antes aludíamos, el contenido de la moral debería enseñarse a través de cualquier método que tenga sentido para el niño en la etapa de desarrollo moral en la que se encuentre, porque, como el propio Peters dice, «En la educación moral, como en cualquier otra forma de educación, los padres y profesores deberán empezar por donde se encuentren los niños $»^{29}$. Por tanto, esperar a que el niño alcance un estadio de desarrollo moral adecuado para poder utilizar las técnicas de estimulación cognoscitiva propuestas por Kohlberg, basadas sobre todo en la discusión, supone una renuncia a la acción educativa temprana, relegando la educación moral a las etapas racionales y descuidando la estimulación necesaria para llegar a ellas, y esto es algo que Peters no acepta, sino que para él es necesaria una sensibilización del niño frente a los demás aun antes de que salga de la etapa egocéntrica, ya que sostiene que el aprendizaje temprano tiene una importancia decisiva para el desarrollo posterior, puesto que considera que en el proceso evolutivo cada período tiene su valor en sí mismo y el desarrollo no consiste en un mero abandono de las etapas anteriores, sino, hablando en términos dialécticos, una superación de ellas, completándolas y perfeccionándolas; dándoles, en definitiva, una significación distinta.

De ahí que, como dice el profesor Santolaria ${ }^{30}$, si el niño es agente mucho antes de ser un agente consciente, han de formarse sus disposiciones para actuar incluso antes de que comprenda las razones para actuar, a modo de fase previa (temporal y lógicamente) del proceso de formación de una persona autónoma. Así, mientras afirma que cuando los niños ya se acercan a la etapa autónoma han de emplearse métodos que contribuyan a estimular su desarrollo y al mismo tiempo fomenten la asunción de roles necesaria en toda autonomía, como la discusión, el tomar parte en

27. op. cit., p. 127.

28. op. cit., p. 130.

29. R. S. PETERS: Desarrollo moral y educación moral, op. cit., p. 208.

30. Santolaria comparte las críticas de Peters al excesivo formalismo kohlbergiano en su brillante trabajo «Consideraciones sobre la educación moral actual», en JORDAN; SANTOLARIA: La educación moral hoy, op. cit., pp. 135-148. 
actividades de grupo, etc., mantiene que en las etapas anteriores, cuando estos modos racionales no significan aún nada para los niños, no podrán utilizarse todavía estos métodos, sino que deberá influenciarse su conducta de otras maneras, por lo que «deberá darse cierta cantidad de instrucción y se deberán seguir ciertos modelos proporcionados por los progenitores y los niños más grandes... pues la mayoría de ellos presuponen captar complicadas disposiciones sociales» ${ }^{31}$.

Sin embargo, esta instrucción y el ejemplo de los adultos probablemente no sean suficientes, pues, aunque al niño le gusta someterse a las reglas de juego a estas edades, tiene al mismo tiempo unas fuertes inclinaciones a realizar lo contrario de lo que establece la regla. Como consecuencia de esto, además de la insistencia de los adultos y los compañeros en el campo de las reglas, han de utilizarse ciertas «ayudas extrínsecas» que favorezcan la aparición de los hábitos de comportamiento que compondrán un «saco de virtudes básico». El tema de la formación de hábitos en educación moral es una cuestión importante en el pensamiento de Peters, ya que constituye el núcleo de una de las críticas que este autor le hace a Kohlberg, para quien la importancia de los hábitos y los procesos de formación de hábitos son secundarios en todo el proceso educativo, puesto que, según él, este tipo de aprendizaje es a corto plazo, se refiere a situaciones específicas y es reversible, como lo refleja en su obra «Moral Education in the School» ${ }^{31}$.

Esta escasa atención de Kohlberg por los hábitos se debe a que su filosofía moral está basada en principios, como la justicia, muy difíciles de captar por un niño pequeño; pero si en vez de este tipo de virtudes se da prioridad, como Peters lo hace, a otras, tales como el interés por los demás, producto de una concepción más pragmática y social de la moral, la relevancia de este aspecto puede ponerse de manifiesto, puesto que estos son principios más fáciles de percibir por los niños de edades tempranas.

Además, según él, al conceder una escasa importancia al hábito, Kohlberg se está oponiendo a toda una tradición de pensamiento sobre el desarrollo moral que parte de Aristóteles, quien, a pesar de asignar un papel relevante a los factores cognoscitivos en el desarrollo moral, al describir a éste como el surgimiento gradual de la razón práctica, concedió también, como ya se insinuó antes, un papel esencial a los hábitos en la moral, cuando dice, en el libro II de la Ética a Nicómaco, que «nos volvemos justos realizando actos justos, templados realizando actos templados, valientes realizando actos valientes».

Sin embargo, Aristóteles, al enfatizar tanto en el desarrollo del pensamiento y la elección crítica como en la formación de hábitos, desembocó en lo que se ha venido llamando LA PARADOJA DE LA EDUCACIÓN MORAL, que Peters, en su ensayo «Razón y Hábito: la paradoja...» formula a través de la comparación con la distinción entre «lenguaje» $\mathrm{y}$ «literatura» de un tema: los «lenguajes» implican reglas de procedimiento, que permiten la crítica, la evaluación y el desarrollo de la «literatura». En este esquema, la educación moral consiste en iniciar a los individuos en el «lenguaje» para que puedan usarlo de una forma autónoma. Esto se logra, en gran medida, mediante la introducción a la «literatura».

31. En School Review, Vol. 74 (1966), pp. 1-30. 
Así, la paradoja de la educación moral, puede describirse como sigue: «Parece deseable el desarrollo de personas que se comporten de forma racional, inteligente y con un alto grado de espontaneidad; sin embargo, los hechos básicos del desarrollo del niño revelan que, en los años más formativos de tal desarrollo, el niño puede llevar esta forma de vida y no entiende su transmisión adecuada» ${ }^{32}$.

Es decir que, para él, las reglas son algo externo e inmutable, y las explicaciones tienen un menor efecto educativo que otro tipo de estrategias de aprendizaje práctico, entre las que destaca el hábito, entendido como tendencia a actuar de una determinada forma, o sea, como habilidad a través de la habituación, o aprendizaje mediante la familiarización con las cosas y los actos.

La paradoja de la educación moral resulta precisamente de ver, por efecto de una confusión conceptual, una incompatibilidad entre el Hábito y el uso de la Razón; sin embargo, esta paradoja se resuelve para Peters si se mantiene, como él lo hace, que «no existe ninguna contradicción necesaria entre el uso de la inteligencia y la formación de hábitos» ${ }^{33}$, puesto que para aprender las reglas y adquirir el hábito de su observación se requiere el empleo de la inteligencia y de la razón, ya que la conducta y el comportamiento han de adaptarse o variarse según la situación concreta, entrando en juego la intención, el interés por la corrección de lo que se hace, la meticulosidad, la verificación y la consideración de las posibles objeciones.

Así, la paradoja de la educación moral es sólo algo teórico, si se piensa que, a pesar de que un código racional de comportamiento todavía no es entendido por los niños pequeños, éstos «pueden entrar al palacio de la Razón por el pasillo del Hábito y de la Tradición» ${ }^{34}$. Por tanto, si los hábitos no se forman solamente a través de la repetición, sino también en el contexto de una actividad concebida de una forma inteligente, pueden proveer de una base apropiada para la etapa autónoma, de conservación o rechazo de las reglas a causa de la existencia o inexistencia de una justificación para ellas.

Al lado de la instrucción y la formación de hábitos, Peters habla también del uso de apoyos o refuerzos en educación moral, entendidos como auxiliares extrínsecos del aprendizaje, que provean al niño de incentivos para superar sus inclinaciones contrarias a la regla y le ayuden a fortalecer los hábitos adquiridos. Al tratar de ellos, hace referencia a los estudios de Hoffman («Moral Development»), quien sugiere que el refuerzo o los auxiliares positivos (remuneración y aprobación),permiten mejor el aprendizaje moral que los negativos (castigo y reprobación), ya que la tensión que éstos crean impide u obstaculiza el aprendizaje de este tipo.

Como dice Peters, «las pruebas demuestran que para que los niños desarrollen una forma autónoma de moralidad requieren un patrón congruente de reglas desde sus primeros años, respaldadas por la aprobación cuando las cumplen ${ }^{35}$, 0 , como apunta más adelante, «de igual manera se puede desquiciar el desarrollo mediante el empleo de técnicas punitivas y de rechazo, que crean ansiedad, la cual a su vez im-

32. R. S. PETERS: Desarrollo moral y educación moral, op. cit., p. 62.

33. R. S. Peters: op. cit., p. 71.

34. R. S. Peters: op. cit., p. 63.

35. R. S. PETERS: «Forma y contenido...», op. cit., p. 131. 
pide el aprendizaje y también mina el desarrollo de la confianza en sí o en los demás» ${ }^{36}$.

Así pues, Peters recomienda la instrucción y la aprobación más que el uso de técnicas de afirmación del poder. Tampoco la tolerancia completa, según él, es aconsejable, ya que frena el desarrollo al crear una ansiedad producida por la ausencia de expectativas, las cuales necesita el niño para prever las consecuencias de sus acciones.

A las críticas que podría suscitar su teoría de que en las primeras etapas de desarrollo moral hay que emplear técnicas de refuerzo, de que hay que atraer a los niños hacia la conformidad con las reglas aun sin que se aprecien las razones para ello, calificando los métodos por los que Peters aboga de «adoctrinamiento», este autor responde que su método de «instrucción moral» no es adoctrinamiento, ya que no incapacita al niño para adoptar una actitud autónoma y crítica hacia las reglas, preguntando además: «¿De qué otra forma se puede proceder? Si desde sus primeros años los niños no pueden adquirir las reglas porque no les ven la razón, y si por los motivos explicados no pueden partir de algún 'saco de virtudes', es difícil ver qué otras alternativas quedan ${ }^{37}$. Dicho de otro modo, ya que a una edad temprana no se puede pensar de una manera autónoma o auténtica en las reglas, tampoco puede hablarse de adoctrinamiento o de inautenticidad a la hora de aplicar estos métodos en educación moral.

Contra este «horror al adoctrinamiento» que invade a muchos padres y educadores, Peters dice: «Así pues, el problema central de los métodos de educación moral temprana puede plantearse de la siguiente manera: dado que se considera deseable que los niños desarrollen una forma autónoma de moralidad y dado que, si Piaget y Kohlberg tienen razón, no puede aprender en los primeros años de un modo que presupone tal forma, ¿cómo se puede proporcionar un contenido básico de moralidad que les dé un fundamento firme de conducta moral, sin impedir el desarrollo de una forma racional para el mismo? ¿Qué métodos no racionales de enseñanza ayudan o siquiera no impiden el desarrollo de la racionalidad? Éste es el problema de la educación moral temprana» ${ }^{38}$. A través de sus planteamientos en el plano teórico, Peters abre, con estos interrogantes, un camino a la investigación en el campo de las metodologías que debe emplear la educación moral durante la enseñanza básica, investigación que urge realizar si se pretende llevar a cabo una práctica educativa eficaz, con miras a lograr el objetivo último de la educación moral, que es, no tanto el desarrollo de «formas» $\mathrm{y}$ «modelos» de razonamiento cuanto el cultivo de la conducta y la acción morales del sujeto, que son, en definitiva, las únicas muestras visibles de su moralidad.

La concepción petersiana de la moral es, por tanto, la de una acción. Y por eso todas sus críticas apuntan a una única, que se puede formular como una denuncia de la simplificación que supone el excesivo énfasis del elemento formal; éste, indudablemente, da a la acción moral el componente racional, autónomo, significativo y crítico, pero no atiende al que debe ser considerado como el interés principal de la

36. R. S. PeTERS: op. cit., p. 132.

37. Ibidem.

38. Ibidem, pp. 133-134. 
educación en este campo: «¿Cómo lograr que los alumnos quieran ser morales?». Y esta voluntad han de empezar a sentirla los niños desde los comienzos del proceso educativo, sin esperar a que por ellos mismos lleguen a alcanzarla. Así, pese a que el esquema de Peters se limite a abordar esta problemática en el campo especulativo, cuando menos deja marcada la idea de que esta educación moral inicial o temprana es ya una tarea irrenunciable. 